\title{
BMJ Open Postnatal exercise interventions: a systematic review of adherence and effect
}

\author{
Edward Mullins (D) , ${ }^{1,2,3}$ Shalini Sharma, ${ }^{4}$ Alison H McGregor ${ }^{5}$
}

To cite: Mullins E, Sharma S, McGregor AH. Postnatal exercise interventions: a systematic review of adherence and effect. BMJ Open 2021;11:e044567. doi:10.1136/ bmjopen-2020-044567

- Prepublication history and additional supplemental material for this paper are available online. To view these files, please visit the journal online (http://dx.doi.org/10.1136/ bmjopen-2020-044567)

Received 07 September 2020 Accepted 20 August 2021
Check for updates

(c) Author(s) (or their employer(s)) 2021. Re-use permitted under CC BY-NC. No commercial re-use. See rights and permissions. Published by BMJ.

${ }^{1}$ Department of Metabolism, Surgery and Cancer, Imperial College London Faculty of Medicine, London, UK ${ }^{2}$ Obstetrics and Gynaecology, Imperial College Healthcare NHS

Trust, London, UK

${ }^{3}$ The George Institute for global health, London, UK

${ }^{4}$ Leicester Royal Infirmary, Leicester, UK

${ }^{5}$ Surgery, Imperial College London Faculty of Medicine,

London, UK

Correspondence to

Edward Mullins;

edward.mullins@ic.ac.uk

\section{ABSTRACT}

Objective To evaluate adherence to and effect of postnatal physical activity (PA) interventions.

Design Systematic review of PA intervention randomised controlled trials in postnatal women. The initial search was carried out in September 2018, and updated in January 2021.

Data sources Embase, MEDLINE and Cochrane Central Register of Controlled Trials (CENTRAL) databases, handsearching references of included studies. The 25 identified studies included 1466 postnatal women in community and secondary care settings.

Eligibility criteria Studies were included if the PA interventions were commenced and assessed in the postnatal year.

Data extraction and synthesis Data were extracted using a prespecified extraction template and assessed independently by two reviewers using Cochrane ROB 1 tool.

Results 1413 records were screened for potential study inclusion, full-text review was performed on 146 articles, 25 studies were included. The primary outcome was adherence to PA intervention. The secondary outcomes were the effect of the PA interventions on the studies' specified primary outcome. We compared effect on primary outcome for supervised and unsupervised exercise interventions. Studies were small, median $n=66$ (20-130). PA interventions were highly variable, targets for PA per week ranged from 60 to 275 min per week. Loss to follow-up (LTFU) was higher (14.5\% vs 10\%) and adherence to intervention was lower $(73.6 \%$ vs $86 \%)$ for unsupervised versus supervised studies.

Conclusions Studies of PA interventions inconsistently reported adherence and LTFU. Where multiple studies evaluated PA as an outcome, they had inconsistent effects, with generally low study quality and high risk of bias. Agreement for effect between studies was evident for PA improving physical fitness and reducing fatigue. Three studies showed no adverse effect of PA on breast feeding. High-quality research reporting adherence and LTFU is needed into how and when to deliver postnatal PA interventions to benefit postnatal physical and mental health.

PROSPERO registration number CRD42019114836.

\section{INTRODUCTION}

Physical inactivity is estimated to cause between $6 \%$ and $10 \%$ of the major

\section{Strengths and limitations of this study}

This review considered only physical activity (PA) intervention studies which were compared with a control group with no PA intervention or with advice to be sedentary, to allow evaluation of effect of the PA intervention on the primary outcomes of each study.

- There may be studies which compare PA interventions in groups other than postnatal women which had high adherence and positive or negative effects and will have been excluded from our analysis.

- We considered only PA interventions in the year after delivery, potentially excluding interventions later after delivery which may be appealing to women and effective in improving physical and mental health.

non-communicable diseases of breast and colon cancer, type 2 diabetes and coronary heart disease and $9 \%$ of premature mortality worldwide. ${ }^{1}$ Not surprisingly, physical inactivity is responsible for one in six UK deaths and is estimated to cost the UK $£ 7.4$ billion annually. ${ }^{2}$ In the UK, $42 \%$ of women are estimated to be not active enough to maintain good health. ${ }^{2}$

Adult physical activity (PA) guidelines recommend $150 \mathrm{~min}$ of moderate-to-vigorous intensity physical activity (MVPA) per week; only $67 \%$ of UK women aged 35-44 achieve this with further reductions with age. ${ }^{3}$ Disparity also exists from a young age; the percentage of girls aged 11 achieving the recommended $60 \mathrm{~min}$ of daily MVPA declines from $20 \%$ to $12 \%$ by the age of 15 ; in boys, this falls from $32 \%$ to $25 \%{ }^{4}$

Women's PA levels are low during and after pregnancy. Sixty per cent of women during pregnancy report engaging in no leisure time PA. ${ }^{5}$ Fewer than $13 \%$ of women meet recommended PA levels during pregnancy, and this decreases with each trimester. ${ }^{6}$ PA measured during and after pregnancy is predominately of a low intensity, providing somewhat limited health benefits. It is important to note that postnatal total PA (measured in hours or 
weeks) is similar to that seen in mid pregnancy, but comprises a larger proportion of care-giving activities. ${ }^{7}$

The postnatal time is a difficult period for women to restart or maintain PA. Fatigue from poor sleep and the demands of infant feeding can also contribute to lower PA levels. The Institute of Health Visitors advise waiting for the 6-week postnatal visit with women's general practitioners (GPs) before resuming high-impact exercise. GPs have to assess maternal and child health at this visit, though this visit is generally short and advice given can be extremely variable. ${ }^{89}$ Healthcare professionals need to know which interventions they can best recommend to postpartum women, enabling them to meet recommended levels of PA and optimise their health.

The UK Chief Medical Officers recommend that pregnant and postnatal women undertake 150 min moderate PA per week, and have provided useful infographics which are available as a guide for women and healthcare professionals. ${ }^{10}{ }^{11}$ These are endorsed by the American College of Obstetricians and Gynaecologists' guidelines, who also recommend 20-30 min of PA per day during pregnancy on most or all days of the week, ${ }^{12}$ and briefly describe the postpartum period as an opportune time to gradually resume an exercise routine. Clearly, however, the figures above indicate that this is not being achieved.

To review available studies on postnatal PA interventions, we conducted a systematic review to determine adherence to postnatal PA interventions, and the effect of the interventions on the outcomes investigated in the studies identified. The results would also inform healthcare professionals who advise women on the most efficacious PA interventions for desired effect.

We wished to explore whether adherence to PA interventions would be greater for supervised (in person supervision or with monitoring of PA, eg, a pedometer) versus unsupervised interventions; and that the effects of PA interventions on the primary outcomes of studies would be related to adherence.

\section{METHODS}

\section{Patient and public involvement}

Patient and public involvement (PPI) for a separate study helped to conceive the research question for this study. We did not undertake PPI for this study.

We carried out a systematic review based on a prepublished protocol and reported the findings in accordance with specifications recommended by the per the Preferred Reporting Items for Systematic reviews and Meta-Analyses checklist. ${ }^{13}$

\section{Search strategy and selection criteria}

Embase, MEDLINE and Cochrane Central Register of Controlled Trials (CENTRAL) databases were searched in duplicate as per the search strategies (online supplemental files 1-3), without restrictions on publication date. The references of included articles were also handsearched by the reviewers to highlight further studies 
for potential inclusion. This review was performed in September 2018, and an updated search was carried out in January 2021. The updated literature research identified one additional report ${ }^{14}$ which included the same participants as those reported in Osman et $\mathrm{al}^{15}$.

An initial screen of titles and abstracts was performed independently by two reviewers (SS and EM) to identify articles for further review. Articles were included after full-text review if they were randomised, controlled trials which compared moderate and/or vigorous PA to no PA. This could be in combination with another intervention, for example, a dietary intervention, if a comparison group with the same dietary intervention alone was tested. If more than one intervention was included, it was essential that there was a comparison between exercise and no exercise groups. If there was any ambiguity regarding a study's inclusion, a third reviewer (AHM) decided on eligibility. Where data were missing or incomplete, contact with the corresponding author was made by email.

Where the PA intervention was restricted to pelvic floor exercises, physiotherapy or single exercise sessions, studies were excluded. Studies were excluded from the review if they did not report on original data or were animal studies. Studies were not excluded based on language, study type or publication status. There was no other restriction on the type of PA intervention.

\section{Data analysis}

Two reviewers (SS and EM) extracted the data from the included studies using a prespecified extraction template. Extracted data elements recorded for each study included general information, eligibility criteria, study characteristics (setting, country, year, design, type and duration of PA intervention, sample size), adherence to the intervention and primary outcome measures.

Both reviewers assessed risk of bias for each study independently using the Cochrane Collaboration's tool for assessing risk of bias. ${ }^{16}$ The following domains were assessed as having a high, low or unclear risk of bias: random sequence generation, allocation concealment, blinding of participants/personnel, blinding of outcome, incomplete outcome, selective reporting and overall bias. The reviewers then used the Grading of Recommendations, Assessment, Development and Evaluations (GRADE) criteria $^{13}$ to assess the quality of the evidence within the review for the specific outcomes identified.

\section{RESULTS}

After the initial search (online supplemental files 1-3), 1413 records were screened for potential study inclusion. After title and abstract review, full-text review was performed on 146 articles. Of these, 25 articles reporting the effect of randomised controlled trials of postnatal exercise interventions versus no exercise were included (table 1). Study designs included control versus exercise; control versus dietary intervention versus dietary intervention and exercise and control versus dietary intervention versus exercise intervention versus dietary and exercise intervention (table 1 ). The 25 studies included 1466 postnatal women.

Studies included were conducted in North America, Europe, Africa, Asia and Australasia. Studies were generally small, ranging from 20 to 130 participants, median $\mathrm{n}=66$. PA interventions used were varied, including provision of active video games (WiiFit and balance board ${ }^{17}$ ), exercise prescriptions, ${ }^{18-22}$ CDs and DVDs,${ }^{23-25}$ telephone ${ }^{26}{ }^{27}$ and face-to-face counselling sessions, ${ }^{27-31}$ group classes $^{24} 253233$ and supervised exercise sessions. ${ }^{14} 15334$ Interventions varied in duration between 11 days and 10 months. Where reported, the targets for PA per week ranged from 60 to 275 min per week. One study prescribed exercise to attain an individualised energy deficit. Four studies did not report a PA target. A meta-analysis was not performed due to the heterogeneity of the studies identified.

Of the studies included, 9 used unsupervised exercise interventions, ${ }^{17212224-273132}$ and 16 used exercise with some form of supervision. Six studies used PA monitoring (a pedometer or an Actigraph), ${ }^{18} 1929303536$ one used PA monitoring and in-person supervised exercise interventions, ${ }^{20}$ six used in-person supervised interventions, ${ }^{14153334} 37-39$ and two used a mixture of in-person supervised and unsupervised interventions ${ }^{2328}$ (One study used an actigraph for 7 days prior to follow-up 6 month post intervention though not during the intervention. ${ }^{23}$ )

Loss to follow-up (LTFU)in the exercise intervention group was reported in 17/26 studies ${ }^{18} 1921-2326-36$ and adherence to the PA intervention was reported by $11 / 26$ studies. ${ }^{18} 2021272931-333639$ When reported, for the groups undergoing PA interventions, mean LTFU was $11.8 \%$ and mean adherence was $80.4 \%$. Some studies reported only overall rates of LTFU and adherence. In studies where LTFU and adherence is reported, the proportions were similar for intervention and control/comparison groups.

Effect of PA intervention on weight loss was reported by $2 / 6$ studies, ${ }^{1732}$ with weight loss between 2.1 and $5.6 \mathrm{~kg}$ reported (table 2). In studies which compared dietary, PA and combined interventions, PA interventions were not associated with weight loss over and above the effect of the dietary intervention. ${ }^{18}{ }^{35}$ Higher rates of LTFU and lower rates of adherence (where reported) were seen in studies which showed an effect on weight loss.

For measures of postnatal depression, $3 / 7$ reported an effect of a PA intervention. ${ }^{20} 2327$ The effect size varied greatly, from -4.79 to -12.8 points on the Edinburgh Postnatal depression scale. 2 out of 3 of these studies reported LTFU, $8 \%$ in both; adherence was reported by $2 / 3$ and was $64 \%$ and $87 \%$.

Physical fitness, defined by $\mathrm{VO}_{2} \max$ and investigated by two small studies, is improved by PA interventions. ${ }^{37} 38$ General fatigue, part of the Multidimensional Fatigue Inventory, appears to be reduced by PA interventions. ${ }^{195}$ Lactation is not impaired by PA interventions in studies with $90 \%$ and $95 \%$ adherence, ${ }^{36}{ }^{39}$ nor is infant growth. ${ }^{34}$ Glycaemia $^{22}$ and sleep quality ${ }^{24}$ appear to be improved 


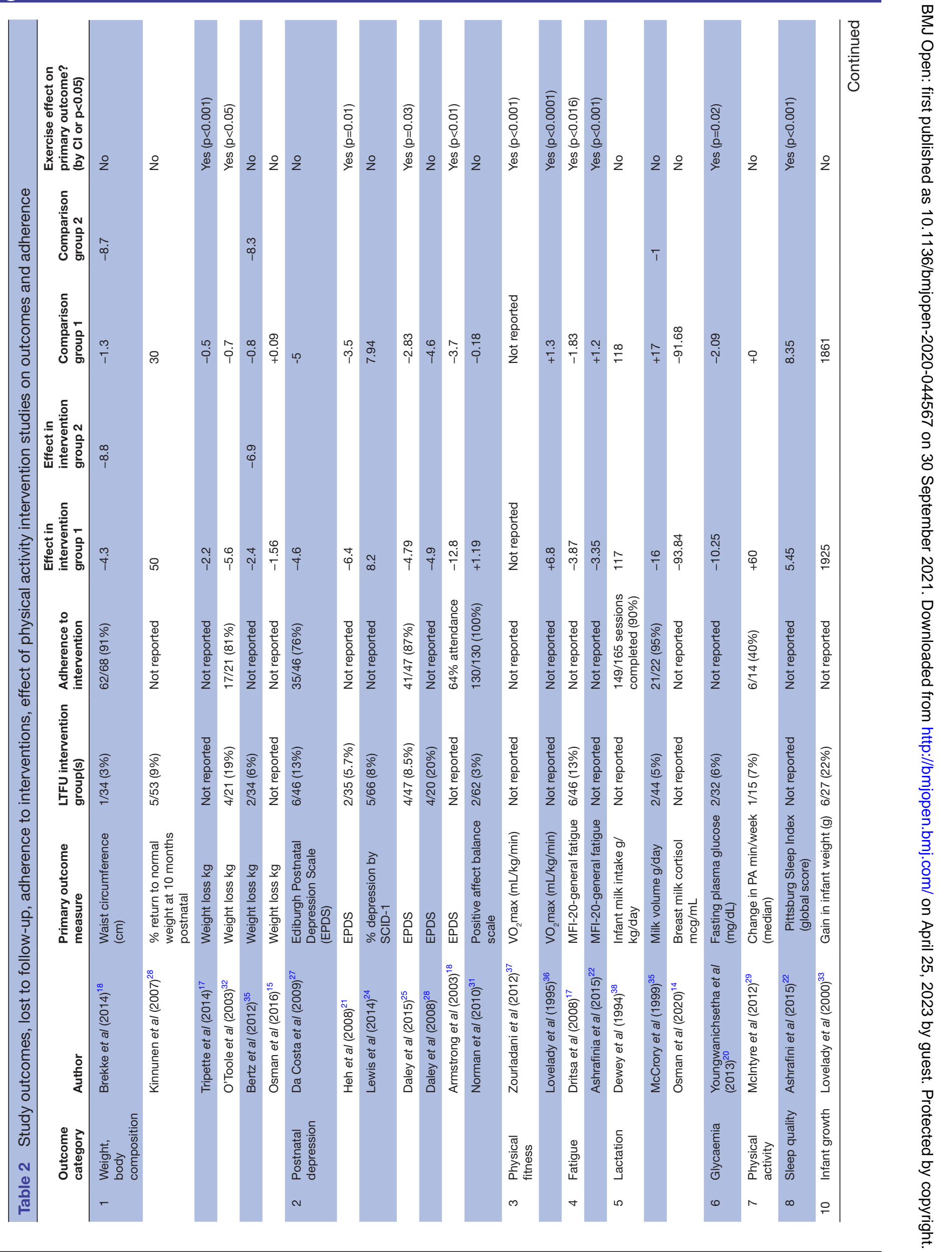




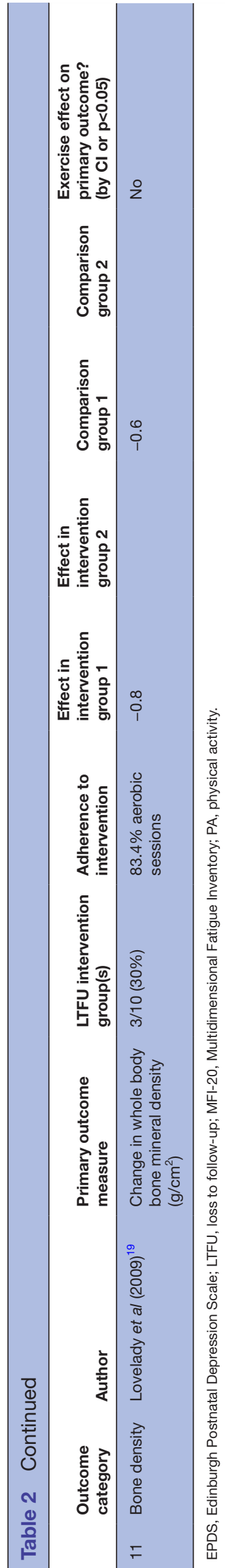

by PA interventions. Bone density, ${ }^{21}$ breast milk cortisol ${ }^{14}$ and (surprisingly) $\mathrm{PA}^{31}$ were not significantly affected by PA interventions, although the latter study had $n=29$ and an adherence of $40 \%$ to the intervention.

Risk of bias assessment found that most studies were at high $(7 / 25)$ or unclear $(16 / 25)$ risk of bias (online supplemental file 1, table 3). Blinding measures for personnel and participants were absent for all studies, conceding that it would be difficult to do so, especially for supervised exercise interventions. No studies referred to a published protocol and/or was convincing that the published report included all expected outcomes, including those that were prespecified. Many studies did not list a primary outcome, in these cases, we used the title or outcome reported first or with most detail in the results section.

Study quality for the various outcomes tested was generally low or very low by GRADE score (table 3), meaning we had low or very low confidence in the effect estimates for all outcomes.

\section{Unsupervised and supervised studies}

Of total, 6/9 unsupervised intervention studies ${ }^{172224252732}$ and 5/16supervisedinterventionstudies ${ }^{141920283738}$ showed an effect of the PA intervention tested on their specified primary outcome (table 4.) LTFU was higher $(14.5 \%$ vs $10 \%)$ and adherence to the intervention was lower $(73.6 \%$ vs $86 \%$ ) for unsupervised versus supervised studies.

\section{DISCUSSION}

In this systematic review, we show that PA interventions have inconsistent effects on most outcomes, with generally low study quality and high risk of bias. Adherence was reported by only $9 / 25$ studies and LTFU for $16 / 25$ studies. For any intervention, the LTFU and adherence must be reported to allow judgement on whether the intervention was acceptable to participants and whether there can be confidence in the effect, or lack of it, shown.

Adjustment for known confounders, such as preexisting exercise habits, physical fitness, weight, BMI were either not present or unclear in the majority of studies. This could have contributed to the heterogeneity of study conclusions.

Supervised exercise interventions did not appear to be more likely to show effect on the various outcomes tested than unsupervised interventions. Studies evaluating unsupervised interventions had lower LTFU and higher adherence, which increases our confidence in the outcomes of the studies. This was unexpected and may reflect that women may feel more empowered when allowed to exercise on their own terms; self-efficacy may lead to greater efficacy of the intervention.

Agreement for effect on primary outcomes in $\geq 2$ studies was evident for PA interventions improving physical fitness $^{3738}$ and reducing fatigue. ${ }^{19}{ }^{25}$ Single studies were identified which reported that PA improved glycaemia ${ }^{22}$ and sleep quality. ${ }^{25}$ These findings are consistent with 


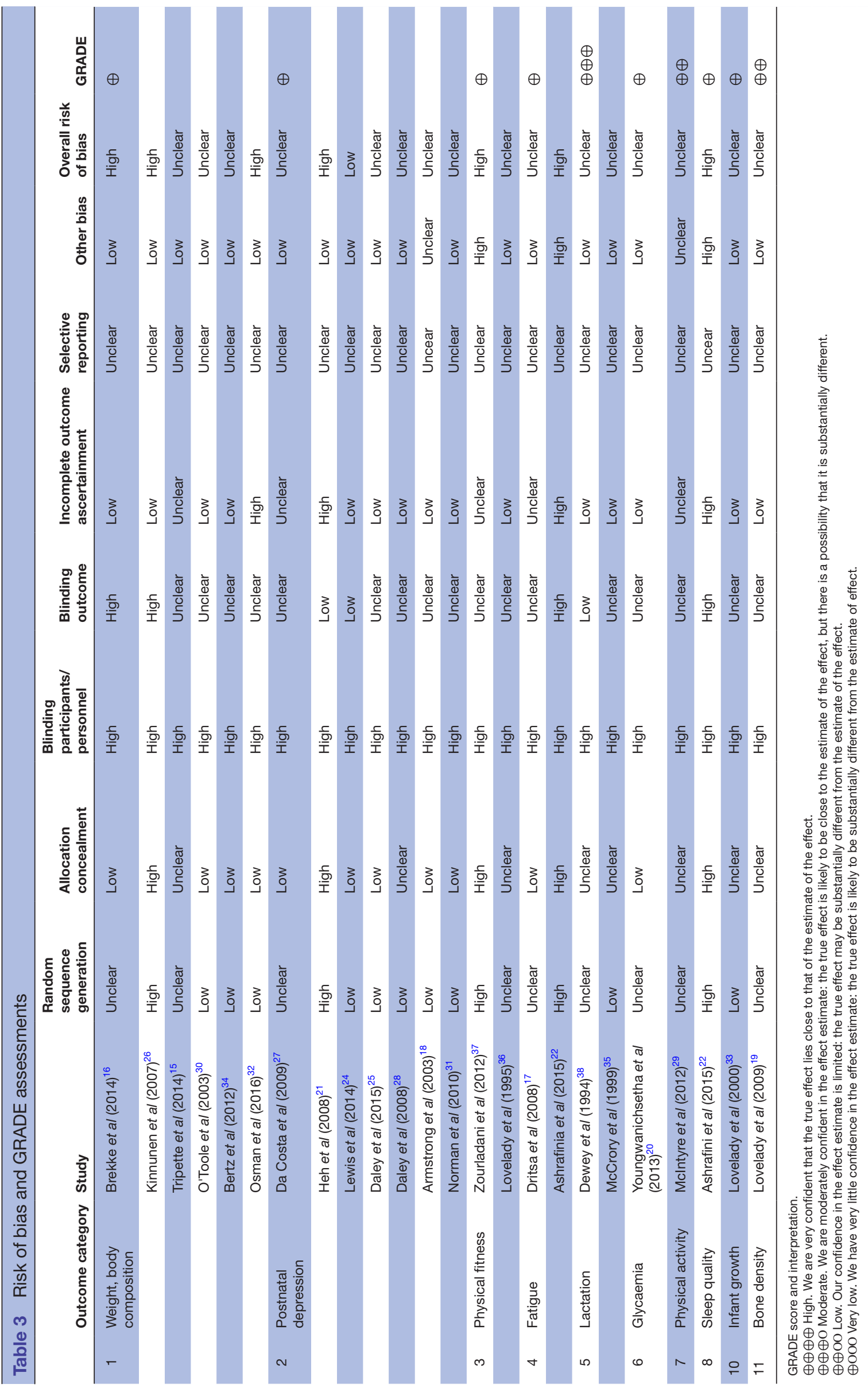


Table 4 Comparison of studies using unsupervised and supervised interventions for mean \% lost to follow-up (LTFU), mean $\%$ adherence and effect of interventions on primary outcome

\begin{tabular}{|c|c|c|c|c|}
\hline Study & $\begin{array}{l}\text { Supervised/ } \\
\text { monitored/ } \\
\text { unsupervised }\end{array}$ & $\begin{array}{l}\text { LTFU intervention } \\
\text { group(s) }\end{array}$ & $\begin{array}{l}\text { Adherence to } \\
\text { intervention }\end{array}$ & $\begin{array}{l}\text { Exercise effect on } \\
\text { primary outcome? } \\
\text { (by } \mathrm{Cl} \text { or } \mathrm{p}<0.05 \text { ) }\end{array}$ \\
\hline \multicolumn{5}{|l|}{ Unsupervised studies } \\
\hline Tripette et al $(2014)^{17}$ & Unsupervised & Not reported & Not reported & Yes $(p<0.001)$ \\
\hline O'Toole et al (2003) $)^{32}$ & Unsupervised & $4 / 21(19 \%)$ & $17 / 21(81 \%)$ & Yes $(p<0.05)$ \\
\hline Lewis et al (2014) & Unsupervised & $5 / 66(8 \%)$ & Not reported & No \\
\hline Daley et al $(2015)^{27}$ & Unsupervised & $4 / 47(8.5 \%)$ & $41 / 47(87 \%)$ & Yes $(p=0.03)$ \\
\hline $\begin{array}{l}\text { Youngwanichsetha et al } \\
(2013)^{22}\end{array}$ & Unsupervised & $2 / 32(6 \%)$ & Not reported & Yes $(p=0.02)$ \\
\hline Ashrafinia et al $(2014)^{24}$ & Unsupervised & Not reported & Not reported & Yes $(p<0.001)$ \\
\hline McIntyre et al (2012) ${ }^{31}$ & Unsupervised & $1 / 15(7 \%)$ & $6 / 14(40 \%)$ & No \\
\hline Ashrafini et al (2015) & Unsupervised & Not reported & Not given & Yes $(p<0.001)$ \\
\hline \multirow[t]{2}{*}{ Lovelady et al (2009) ${ }^{21}$} & Unsupervised & $3 / 10(30 \%)$ & $83.4 \%$ aerobic sessions & No \\
\hline & & $\begin{array}{l}14.5 \% \text { (reported by } \\
6 / 9 \text { ) }\end{array}$ & $73.6 \%$ (reported by $4 / 9$ ) & $6 / 9$ showed effect \\
\hline \multicolumn{5}{|l|}{ Supervised studies } \\
\hline Brekke et al $(2014)^{18}$ & HR monitor & $1 / 34(3 \%)$ & $62 / 68(91 \%)$ & No \\
\hline Bertz et al (2012) $)^{35}$ & HR monitor & $2 / 34(6 \%)$ & Not reported & No \\
\hline Da Costa et al (2009) $)^{29}$ & HR monitor & $6 / 46(13 \%)$ & $35 / 46(76 \%)$ & No \\
\hline Dritsa et al $(2008)^{19}$ & HR monitor & $6 / 46(13 \%)$ & Not reported & Yes $(p=0.016)$ \\
\hline McCrory et al (1999) & HR monitor & $2 / 44(5 \%)$ & $21 / 22(95 \%)$ & No \\
\hline Daley et al (2008) & Pedometer & $4 / 20(20 \%)$ & Not reported & No \\
\hline Kinnunen et al $(2007)^{28}$ & $\begin{array}{l}\text { Unsupervised, optional } \\
\text { supervised }\end{array}$ & $5 / 53(9 \%)$ & Not reported & No \\
\hline Heh et al $(2008)^{23}$ & $\begin{array}{l}\text { Supervised and } \\
\text { unsupervised }\end{array}$ & $2 / 35(5.7 \%)$ & Not reported & Yes $(p=0.01)$ \\
\hline Osman et al $(2016)^{15}$ & Supervised & Not reported & Not reported & No \\
\hline Osman et al $(2020)^{14}$ & Supervised & Not reported & Not reported & No \\
\hline Armstrong et al (2003) & Supervised, Pedometer & Not reported & $64 \%$ attendance & Yes $(p<0.01)$ \\
\hline Norman et al $(2010)^{33}$ & Supervised & $2 / 62(3 \%)$ & $130 / 130(100 \%)$ & No \\
\hline Zourladani et al (2012) ${ }^{37}$ & Supervised & Not reported & Not reported & Yes $(p<0.001)$ \\
\hline Lovelady et al (1995) & Supervised & Not reported & Not reported & Yes $(p<0.0001)$ \\
\hline Dewey et al (1994) & Supervised & Not reported & $\begin{array}{l}149 / 165 \text { sessions } \\
\text { completed }(90 \%)\end{array}$ & No \\
\hline \multirow[t]{2}{*}{ Lovelady et al (2000) } & Supervised & $6 / 27(22 \%)$ & Not given & No \\
\hline & & $\begin{array}{l}9.97 \% \text { (reported by } \\
10 / 16 \text { ) }\end{array}$ & $86 \%$ (reported by 6/16) & $5 / 16$ showed effect \\
\hline
\end{tabular}

HR, heart rate; LTFU, lost to follow-up.

changes in physiology which would be expected with increased PA and as seen in other populations. ${ }^{40} 41$

Three studies showed no effect of physical activity interventions on breast milk production, intake and cortisol levels, ${ }^{14} 3639$ which is encouraging for women who wish to undertake recommended PA levels in the postnatal period and breastfeed.

Women considering postnatal PA can be counselled that PA is unlikely to have an impact on breastfeeding or infant growth. Additionally, no studies led to any serious adverse events. While the wide range of interventions makes recommendations on the types of exercise difficult to assess, it also demonstrates the inventiveness of researchers in the field who have considered using video games, ${ }^{17}$ pilates, ${ }^{24}{ }^{25}$ tai chi ${ }^{22}$ and telephone-based interventions ${ }^{26}$ in addition to more conventional exercise counselling and supervised exercise classes. 
Since the majority of these studies were conducted, there are now many wearable devices available, which could improve objective assessment of PA in intervention studies. These will allow accurate assessment of adherence and measures of fitness to be measured, such as morning resting heart rate. Our group is conducting feasibility studies before, during and after pregnancy using wearables and phone applications to monitor cardiovascular health.

Strengths of this study included a published protocol and a literature search which was updated close to publication. In order to provide information relevant to clinicians and policy-makers looking to improve PA in postnatal women, we focused our study on moderatevigorous exercise programmes which were evaluated for effect on a range of mental and physical health parameters. A key factor in designing and costing PA interventions is whether they are supervised or unsupervised, our study allows these groups to be compared. The weakness of this study is that the studies included were generally small in size and of largely low quality and high or uncertain risk of bias. This review considered only PA intervention studies which were compared with a control group with no PA intervention or with advice to be sedentary, to allow evaluation of effect of the PA intervention on the primary outcomes of each study. Our inclusion of only women in the postnatal year meant that we did not included studies which compares PA interventions in groups other than postnatal women which had high adherence and positive or negative effects and will have been excluded from our analysis. Inclusion of only PA interventions in the year after delivery may also have potentially excluded studies of interventions later after delivery which may be appealing to women and effective in improving physical and mental health.

Further research is needed on the optimal timing, method of delivery and content of postnatal PA interventions to maximise recruitment, adherence and ensure effects on outcomes are effectively evaluated. Weight gain is encouraged in pregnancy, but complications such as excessive gestational weight gain, preeclampsia or gestational diabetes may help identify women likely to benefit from PA interventions to improve their long-term health. Studies must report using internationally agreed standards and outcome sets to improve their utility to women and their healthcare professionals.

More generally, the postnatal period is a difficult time restart and maintain PA. Public health approaches which encourage and enable women to increase their PA after having a baby could have broader population impacts with benefits seen in short-term and long-term risk of chronic diseases. However, studies to date have not consistently demonstrated these effects. None of the included studies referred to public and patient involvement (PPI) in the design or conduct of their research. PPI is invaluable to ensure recruitment, adherence and rigorous evaluation with outcomes which are relevant to women and their healthcare providers.
Contributors EM, SS and AHM were involved in study design and conduct, data analysis, report drafting and editing. EM and SS conducted the literature review.

Funding The authors have not declared a specific grant for this research from any funding agency in the public, commercial or not-for-profit sectors.

Competing interests None declared.

Patient consent for publication Not required.

Provenance and peer review Not commissioned; externally peer reviewed.

Data availability statement Data are available upon reasonable request.

Supplemental material This content has been supplied by the author(s). It has not been vetted by BMJ Publishing Group Limited (BMJ) and may not have been peer-reviewed. Any opinions or recommendations discussed are solely those of the author(s) and are not endorsed by BMJ. BMJ disclaims all liability and responsibility arising from any reliance placed on the content. Where the content includes any translated material, BMJ does not warrant the accuracy and reliability of the translations (including but not limited to local regulations, clinical guidelines, terminology, drug names and drug dosages), and is not responsible for any error and/or omissions arising from translation and adaptation or otherwise.

Open access This is an open access article distributed in accordance with the Creative Commons Attribution Non Commercial (CC BY-NC 4.0) license, which permits others to distribute, remix, adapt, build upon this work non-commercially, and license their derivative works on different terms, provided the original work is properly cited, appropriate credit is given, any changes made indicated, and the use is non-commercial. See: http://creativecommons.org/licenses/by-nc/4.0/.

ORCID iD

Edward Mullins http://orcid.org/0000-0003-1886-6358

\section{REFERENCES}

1 Lee I-M, Shiroma EJ, Lobelo F, et al. Effect of physical inactivity on major non-communicable diseases worldwide: an analysis of burden of disease and life expectancy. Lancet 2012;380:219-29.

2 Public Health England. Physical activity: applying All Our Health [Internet]. Available: https://www.gov.uk/government/publications/ physical-activity-applying-all-our-health/physical-activity-applyingall-our-health [Accessed 08 Aug 2019].

3 Scholes S. Health survey for England 2016 physical activity in adults. NHS Digital, 2017: 20. https://files.digital.nhs.uk/publication/m/3/ hse16-adult-phy-act.pdf

4 Currie C, Zanotti C, Morgan A. Social determinants of health and well-being among young people. health behaviour in school-aged children (HBSC) study: international report from the 2009/2010 survey. World Heal Organ Heal Policy Child Adolesc 2012;6:1-272 http://www.euro.who.int/en/health-topics/Life-stages/child-andadolescent-health/publications/2012/social-determinants-of-healthand-well-being-among-young-people.-health-behaviour-in-schoolaged-children-hbsc-study

5 Hesketh KR, Evenson KR. Prevalence of U.S. pregnant women meeting 2015 ACOG physical activity guidelines. Am J Prev Med 2016;51:e87-9.

6 Borodulin KM, Evenson KR, Wen F, et al. Physical activity patterns during pregnancy. Med Sci Sports Exerc 2008;40:1901-8.

7 Borodulin K, Evenson KR, Herring AH. Physical activity patterns during pregnancy through postpartum. BMC Womens Health 2009;9:1-7.

8 National Childbirth Trust. NCT \& Netmums research finds the six week postnatal check-up unsatisfactory [Internet], 2014. Available: https://www.nct.org.uk/about-us/news-and-views/news/nctnetmums-research-finds-six-week-postnatal-check-unsatisfactory

9 Davies SC. Annual Report of the Chief Medical Officer [Internet]. Annual Report of the Chief Medical Officer. The Health of the 51\%: Women, 2014. Available: https://www.gov.uk/government/ organisations/department-of-health

10 Department of Health and Social Care. Physical activity for pregnant women [Internet]. Promotional material Start active, stay active: infographics on physical activity, 2017. Available: https://assets. publishing.service.gov.uk/government/uploads/system/uploads/ attachment_data/file/622335/CMO_physical_activity_pregnant_ women infographic.pdf

11 Physical activity for women after childbirth 2019;12.

12 Committee on Obstetric Practice. Committee opinion no. 650: physical activity and exercise during pregnancy and the postpartum period. Obstet Gynecol 2015;126:135-42. 
13 Page MJ, McKenzie JE, Bossuyt PM, et al. The PRISMA 2020 statement: an updated guideline for reporting systematic reviews. BMJ 2021;372:n71.

14 Osman DA, Yousef AM, El-badry S. Impact of moderate exercise on breast milk cortisol in healthy lactating women: a randomized controlled trial. Eurasian Journal of Biosciences 2020;14:1113-7.

15 Osman D, Yousef A, El-Badry S. Effect of moderate exercise on breast milk leukocytes in exclusively breast-feeding mothers. Int $J$ PharmTech Res 2016;9:01-8.

16 Green S, Higgins J. Cochrane Handbook for Systematic Reviews of Interventions v5.1.0 [Internet]. 2011. p. Section 8.5. Available: https:// handbook-5-1.cochrane.org/chapter_8/table_8_5_a_the_cochrane_ collaborations_tool_for_assessing.htm

17 Tripette J, Murakami H, Gando Y, et al. Home-Based active video games to promote weight loss during the postpartum period. Med Sci Sports Exerc 2014;46:472-8.

18 Brekke HK, Bertz F, Rasmussen KM, et al. Diet and exercise interventions among overweight and obese lactating women: randomized trial of effects on cardiovascular risk factors. PLoS One 2014;9:e88250.

19 Dritsa M, Da Costa D, Dupuis G, et al. Effects of a home-based exercise intervention on fatigue in postpartum depressed women: results of a randomized controlled trial. Ann Behav Med 2008;35:179-87.

20 Armstrong K, Edwards $\mathrm{H}$. The effects of exercise and social support on mothers reporting depressive symptoms: a pilot randomized controlled trial. Int J Ment Health Nurs 2003;12:130-8.

21 Lovelady CA, Bopp MJ, Colleran HL, et al. Effect of exercise training on loss of bone mineral density during lactation. Med Sci Sports Exerc 2009;41:1902-7.

22 Youngwanichsetha S, Phumdoung S, Ingkathawornwong T. The effects of tai chi qigong exercise on plasma glucose levels and health status of postpartum Thai women with type 2 diabetes. Focus on Alternative and Complementary Therapies 2013;18:182-7.

23 Heh S-S, Huang L-H, Ho S-M, et al. Effectiveness of an exercise support program in reducing the severity of postnatal depression in Taiwanese women. Birth 2008;35:60-5

24 Ashrafinia F, Mirmohammadali M, Rajabi H, et al. The effects of Pilates exercise on sleep quality in postpartum women. J Bodyw Mov Ther 2014;18:190-9.

25 Ashrafinia F, Mirmohammadali M, Rajabi H, et al. Effect of Pilates exercises on postpartum maternal fatigue. Singapore Med $J$ 2015;56:169-73.

26 Lewis BA, Gjerdingen DK, Avery MD, et al. A randomized trial examining a physical activity intervention for the prevention of postpartum depression: the healthy mom trial. Ment Health Phys Act 2014;7:42-9.

27 Daley AJ, Blamey RV, Jolly K, et al. A pragmatic randomized controlled trial to evaluate the effectiveness of a facilitated exercise intervention as a treatment for postnatal depression: the PAM-PeRS trial. Psychol Med 2015;45:2413-25.

28 Kinnunen TI, Pasanen M, Aittasalo M, et al. Reducing postpartum weight retention - a pilot trial in primary health care. Nutr $J$ 2007;6:1-9.

29 Da Costa D, Lowensteyn I, Abrahamowicz M, et al. A randomized clinical trial of exercise to alleviate postpartum depressed mood. J Psychosom Obstet Gynaecol 2009;30:191-200.

30 Daley A, Winter H, Grimmett C, et al. Feasibility of an exercise intervention for women with postnatal depression: a pilot randomised controlled trial. Br J Gen Pract 2008;58:178-83.

31 Mclntyre HD, Peacock A, Miller YD, et al. Pilot study of an individualised early postpartum intervention to increase physical activity in women with previous gestational diabetes. Int $\mathrm{J}$ Endocrinol 2012;2012:1-5.

32 O’toole ML, Sawicki MA, Artal R. Structured Diet and Physical Activity Prevent Postpartum Weight Retention [Internet]. Vol. 12, JOURNAL OF WOMEN'S HEALTH, 2003. Available: www.liebertpub. com [Accessed cited 2018 Aug 21].

33 Norman E, Sherburn M, Osborne RH, et al. An exercise and education program improves well-being of new mothers: a randomized controlled trial. Phys Ther 2010;90:348-55.

34 Lovelady CA, Garner KE, Moreno KL, et al. The effect of weight loss in overweight, lactating women on the growth of their infants. $N$ Engl J Med 2000;342:449-53.

35 Bertz F, Brekke HK, Ellegård L, et al. Diet and exercise weight-loss trial in lactating overweight and obese women. Am J Clin Nutr 2012;96:698-705.

36 McCrory MA, Nommsen-Rivers LA, Molé PA, et al. Randomized trial of the short-term effects of dieting compared with dieting plus aerobic exercise on lactation performance. Am J Clin Nutr 1999;69:959-67.

37 Zourladani A, Zafrakas M, Chatzigiannis B, et al. The effect of physical exercise on postpartum fitness, hormone and lipid levels: a randomized controlled trial in primiparous, lactating women. Arch Gynecol Obstet 2015;291:525-30.

38 Lovelady CA, Nommsen-Rivers LA, McCrory MA, et al. Effects of exercise on plasma lipids and metabolism of lactating women. Med Sci Sports Exerc 1995;27:22-8.

39 Dewey KG, Lovelady CA, Nommsen-Rivers LA, et al. A randomized study of the effects of aerobic exercise by lactating women on breast-milk volume and composition. N Engl J Med 1994;330:449-53.

40 Canhin DdaS, Tebar WR, Scarabottolo CC, et al. Physical activity across life stages and sleep quality in adulthood - an epidemiological study. Sleep Med 2021;83:34-9.

41 Cramp F, Byron-Daniel J. Exercise for the management of cancer-related fatigue in adults. Cochrane Database Syst Rev 2012;11:CD006145. 